Влияние протокола

антимикробной терапии

на результаты лечения

респираторных инфекций

у пациентов ОРИТ

многопрофильного стационара

\author{
М.Н. Замятин², О.А. Векшина', В.Г. Гусаров², \\ С.У. Кузьминов ${ }^{1}$, И.В. Бардин ${ }^{1}$, П.А. Князева ${ }^{1}$
}

1 ГБУРО «Областная клиническая больница», Рязань, Россия
${ }^{2}$ ФГБУ «НМХЦ им. Н.И. Пирогова» Минздрава России, Москва,
Россия

Реферат

Обоснование. Результаты лечения респираторной инфекции у пациента в отделении реанимации и интенсивной терапии прямо зависят от того, насколько эффективной будет проводимая антимикробная терапия. Одним из способов, рекомендуемых для организации такой терапии, является локальный протокол, основанный на локальных данных об уровне антибиотикорезистентности и предусматривающий ряд действий при проведении диагностики и лечения инфекций, однако данные об эффективности такой формы контроля в различных стационарах противоречивы.

Цель исследования. Оценить эффективность применения локального протокола лечения респираторных инфекций в отделении анестезиологии и реаниматологии (ОАР) многопрофильного стационара.

Материалы и методы. Ретроспективное интервенционное исследование с историческим контролем выполнено в областной клинической больнице на 940 коек. Протокол включал показания для начала терапии, алгоритм выбора и отмены антибиотика. Для анализа использовали данные всех пациентов с пневмонией и гнойным трахеобронхитом, находившихся на лечении в ОАР в течение одного календарного года до и одного года после внедрения протокола (преинтервенционный и интервенционный периоды). В соответствии с критериями диагностики из 1090 пациентов, находившихся на лечении

\section{Effect of the antimicrobial stewardship protocol on the results of treatment of ICU patients with respiratory infections in a multidisciplinary hospital. Article}

\author{
M.N. Zamyatin², O.A. Vekshina', V.G. Gusarov², \\ S.U. Kuzminov ${ }^{1}$, I.V. Bardin¹, P.A. Knyazeva ${ }^{1}$ \\ ${ }^{1}$ Ryazan State medical hospital, Ryazan, Russia \\ ${ }^{2}$ National Medical and Surgical Center named after N.I. Pirogov, \\ Moscow, Russia
}

\section{Abstract}

The purpose of the study was to evaluate the effectiveness of implementation in real practice of multidisciplinary hospital ICU a local Protocol for the treatment of respiratory infections.

Materials and methods. The retrospective interventional study with historical control was performed in a regional clinical hospital with 940 beds. The Protocol was based on the results of local bacteriological data and included indications for start therapy, algorithm for selecting and canceling the antibiotic treatment. We included in analysis data from all patients with pneumonia and purulent tracheobronchitis who were treated in ICU for previous andnext year after the implementation of the Protocol (pre- and intervention period). In accordance with the diagnostic criteria, a group of 146 patients was formed out of 1090 patients who were treated in ICU in preintervention period and to compare with 174 patients group in the interventional period out of 1465 ICU patients.

Results. The implementation of the Protocol helped to reduce the average duration of the AMT course from 13.7 to 10.4 days, $p<0.01$, the frequency of recurrence of pulmonary infection (22.6\% and $12.6 \%, p=0.028$ ), the duration of artificial ventilation $(p<0.01)$ and treatment in ICU (for 3 days, $p=0.025$ ). Overall mortality in the groups decreased insignificantly $(p=0.06)$. 
в ОАР в год перед интервенцией, сформирована группа из 146 больных (13,4%); в интервенционный период из 1465 пациентов ОАР в группу сравнения включили 174 больных (11,9 \%).

Результаты. Внедрение протокола позволило снизить среднюю длительность курса антимикробной терапии с 13,7 до 10,4 дней $(p<0,01)$, частоту рецидивов легочной инфекции (22,6 и 12,6 \%; $p=0,028)$, продолжительность искусственной вентиляции легких $(p<0,01)$ и лечения (на 3 суток; $p=0,025)$, число пациентов, нуждающихся в искусственной вентиляции легких более 48 ч $(93,75$ и 72,5 \%; $p=0,044)$. Общая летальность в группах снизилась статистически незначимо $(p=0,06)$.

Заключение. Локальный протокол лечения респираторных инфекций является действенным инструментом контроля антимикробной терапии. Выполнение протокола позволяет врачу своевременно и обоснованно назначить пациенту те препараты, которые обеспечат раннее начало эффективной терапии, а затем также обоснованно изменить схему или прекратить терапию. Как следствие, улучшаются результаты лечения, снижается вероятность прогрессирования или рецидива инфекции, необходимости перевода пациентов на искусственную вентиляцию легких, сокращается длительность респираторной поддержки и пребывания пациентов в отделении интенсивной терапии.

Ключевые слова: респираторные инфекции, антибиотикорезистентность, контроль антимикробной терапии

凶 Для корреспонденции: Векшина Ольга Андреевна - врач анестезиолог-реаниматолог отделения анестезиологии и реанимации № 1 ГБУ РО «Областная клиническая больница», Рязань; e-mail: olvekshina@yandex.ru

ए Для цитирования: Замятин М.Н., Векшина О.А., Гусаров В.Г., Кузьминов С.У., Бардин И.В., Князева П.А. Влияние протокола антимикробной терапии на результаты лечения респираторных инфекций у пациентов ОРИТ многопрофильного стационара. Вестник интенсивной терапии им. А.И. Салтанова. 2020;2:96-103.

๘ Поступила: 26.01.2020

п. Принята к печати: 02.06.2020
Conclusion. Protocol for respiratory infections treatment is an effective tool for control of antimicrobial therapy. The implementation of the Protocol helps physician to timely and reasonably prescribe those antibiotics to the patient that will ensure an early start of effective therapy, and then also reasonably change the scheme or stop therapy. As a result, treatment results are improved, the probability of progression or recurrence of infection is reduced, the need of ventilatory support is reduced, as well as the duration of respiratory support and stay of patients in the ICU.

Keywords: respiratory infection, antimicrobial resistance, antimicrobial stewardship

$凶$ For correspondence: Olga A. Vekshina, physician anesthesiologist-reanimatologist, Ryazan State medical hospital, Ryazan; e-mail: olvekshina@yandex.ru

¿ For citation: Zamyatin M.N., Vekshina O.A., Gusarov V.G., Kuzminov S.U., Bardin I.V., Knyazeva P.A. Effect of the antimicrobial stewardship protocol on the results of treatment of ICU patients with respiratory infections in a multidisciplinary hospital. Article. Annals of Critical Care. 2020;2:96-103.

¿ Received: 26.01.2020

自 Accepted: 02.06 .2020

\section{Введение}

Хорошо известно, что наличие инфекции у пациента, находящегося в отделении реанимации и интенсивной терапии (ОРИТ), ухудшает прогноз течения заболевания, увеличивает стоимость и длительность лечения, ведет к повышению летальности и инвалидизации [1-3]. Особенно это касается инфекций нижних дыхательных путей и легкого, которые всегда представляют угрозу для жизни больного в критическом состоянии, независимо от того, является ли инфекционный процесс причиной госпитализации или осложнением, 
развившимся в процессе интенсивной терапии. При тяжелом течении внебольничной пневмонии летальность составляет 15-48 \% [4]. Вероятность летального исхода при нозокомиальной пневмонии, не требующей перевода пациента на искусственную вентиляцию легких (ИВЛ), достигает 9,8-21,7 \%; если пневмония вызывает декомпенсацию дыхательной недостаточности, и пациент нуждается в проведении ИВЛ, риск летального исхода повышается до 15,2-39,4 \%, а при пневмонии, ассоциированной с ИВЛ, - до 12,6-27 \% [5, 6]. В свою очередь, летальность при тяжелых инфекциях прямо зависит от того, как быстро начата эффективная антимикробная терапия (AMT) [1, 7]. Это означает, что стартовая AMT, которую обычно называют «эмпирической», должна быть адресной и проводиться с учетом наиболее вероятных возбудителей данной инфекции и предполагаемой чувствительности этих возбудителей к антимикробным препаратам. Методология такого подхода к проведению АМТ детально изложена в российских рекомендациях «Программа СКАТ (Стратегия контроля антимикробной терапии) при оказании стационарной медицинской помощи» $[8,9]$. В соответствии с рекомендациями стратегия может быть реализована несколькими путями, например, с помощью преавторизации назначения антимикробных препаратов или определенной их группы или проспективного аудита с обратной связью, однако такие способы предполагают наличие специальных человеческих, финансовых и информационных ресурсов и направлены в первую очередь на снижение нерационального избыточного потребления антибиотиков без негативного влияния на исходы пациентов с инфекцией. Другой формой контроля являются локальные рекомендации или внутрибольничные протоколы AMT, основанные на данных внутреннего микробиологического мониторинга в стационаре и предусматривающие выполнение ряда последовательных действий при проведении диагностики и лечении инфекций. Такие протоколы, особенно реализованные в виде компьютерных программ, больше соответствуют принципам использования систем поддержки принятия решения, помогающим врачам выбрать верный алгоритм действий и уменьшить вероятность ошибки при проведении АМТ. Снижение расхода препаратов и стоимости AMT, так же как и изменение уровня антибиотикорезистентности, в этом случае носит вторичный опосредованный характер. Эффективность СКАТ и аналогичных программ, получивших в мировой практике общее название “antimicrobial stewardship program”, подтверждена многими российскими и зарубежными исследованиями [10-14], однако в реальной клинической практике реализация таких программ встречается с серьезными трудностями и препятствиями, что особенно заметно при внедрении их в практику крупных многопрофильных стационаров, где в одном ОРИТ могут быть сосредоточены пациенты с самыми разнообразными формами инфекции нижних дыхательных путей и легкого, принципиально отличающимися по этиологии, механизмам развития, течению заболевания, объему и содержанию интенсивной терапии, коморбидному фону [15].

Цель исследования - оценить эффективность применения локального протокола лечения респираторных инфекций в отделении анестезиологии и реаниматологии (ОАР) многопрофильного стационара.

\section{Материалы и методы}

Ретроспективное интервенционное с историческим контролем исследование эффективности локального протокола эмпирической АМТ респираторных инфекций выполнено в условиях областной клинической больницы (ОКБ) на 940 коек в соответствии с программой научно-исследовательской работы, одобренной локальным этическим комитетом. В течение 2015 г. (начало интервенции) в ОАР ОКБ был внедрен протокол эмпирической АМТ, разработанный на основе действующих рекомендаций и анализа результатов первичных бактериологических исследований материала, полученного у 150 пациентов с инфекциями нижних дыхательных путей и легкого, стратифицированных по риску наличия резистентных возбудителей. Все бактериологические исследования выполняли в локальной лаборатории, чувствительность патогенов к антимикробным препаратам определяли диск-диффузионным методом.

В соответствии с целью исследования в сравнительный анализ включили данные всех пациентов с пневмонией (внебольничной, нозокомиальной, вентиляторассоциированной) и острым гнойным трахеобронхитом, находившихся на лечении в ОАР в течение одного календарного года (2014) до внедрения протокола (группа исторического контроля, преинтервенционный период) и одного календарного года (2016), следующего после внедрения протокола (интервенционный период).

В протоколе для стартовой терапии в каждой группе риска были определены те антимикробные препараты (или их комбинации), к которым были чувствительны не менее 80 \% штаммов наиболее вероятных патогенов. Показанием для проведения АМТ стало наличие у пациента клинических и лабораторных признаков инфекционного процесса в легком (6 и более баллов по шкале CPIS). В качестве методов, позволяющих подтвердить диагноз пневмонии (с учетом клинических данных), использовали рентгенографию грудной клетки или компьютерную томографию, при которых выявляли наличие на рентгенограмме «свежих» очагово-инфильтративных изменений в легких. Диагностические критерии внебольничной, нозокомиальной и вентилятор-ассоциированной пневмонии и острого 
трахеобронхита определялись стандартно, по сроку развития инфекции.

Аналогичные критерии постановки диагноза использовали для формирования группы сравнения при ретроспективном анализе медицинских карт пациентов, лечившихся в ОРИТ в преинтервенционном периоде. Таким способом из 1090 пациентов, находившихся на лечении в ОАР в 2014 г. перед интервенцией, была сформирована группа из 146 больных (13,4 \%) с подтвержденным диагнозом инфекции нижних дыхательных путей и легкого. В постинтервенционный период (2016г.) из 1465 пациентов ОРИТ в группу сравнения включили 174 больных $(11,9 \% ; p=0,278)$

Для оценки эффективности протокола эмпирической АМТ использовали следующие показатели:

- средняя длительность курса АМТ при респираторных инфекциях:

общее число дней АМТ у пациентов
с респираторными инфекциями
общее число больных с респираторными
инфекциями

- количество дней АМТ при респираторных инфекциях:

$\frac{\begin{array}{c}\text { общее число дней АМТ у пациентов } \\ \text { с респираторными инфекциями }\end{array}}{\text { общее число пациентов }} \cdot$

Поскольку у части больных АМТ была начата в ОАР и продолжена в профильном отделении, эти показатели рассчитаны в том числе и для общей продолжительности курсов АМТ.

- Частота легочной реинфекции (случаев повторного развития признаков инфекции той же локализации после отмены АМТ):

общее число больных с легочной реинфекцией $\times 100 \%$

общее число пациентов с респираторными инфекциями

- Продолжительность лечения, общая летальность среди пациентов с респираторными инфекциями в ОАР, частота перевода таких пациентов на ИВЛ и продолжительность ИВЛ.

Статистическую обработку полученных данных проводили с использованием статистических модулей электронных таблиц приложения Microsoft Excel 2007 к пакету Microsoft Office 2007 (Microsoft, США) и пакета статистического анализа STATISTICA 7.0 (StatSoft, США). Анализ нормальности распределения изучаемых совокупностей проводился с использованием критерия КолмогороваСмирнова. В части описательной статистики использованы:
- для количественных признаков, имеющих нормальное распределение, - выборочное среднее значение $(\mu) \pm$ выборочное стандартное отклонение $(\delta)$;

- для количественных признаков, не имеющих нормального распределения, - медиана (Me), первый (Q1) и третий квартили (Q3);

- для качественных признаков - абсолютное число и доля $(n, \%)$.

Сравнение количественных признаков в несвязанных совокупностях, имеющих нормальное распределение, проводили с помощью t-критерия Стьюдента (статистическую значимость различий устанавливали с 95\%-м доверительным интервалом $(p<0,05))$. При сравнении количественных признаков, не имеющих нормального распределения, использован U-критерий Манна-Уитни. При сравнении качественных признаков использован расчет критерия $\chi^{2}$ с поправкой Йетса.

\section{Результаты и их обсуждение}

Средний возраст пациентов сравниваемых групп составил $54,8 \pm 16,9$ и 57,1 $\pm 16,6$ года $(p=0,21)$, причины перевода в ОРИТ были разнообразны, при этом отличий в структуре профилей пациентов не выявлено. В обеих группах основными причинами лечения в ОРИТ были черепно-мозговая травма $(21,2$ и $26,6 \% ; p=0,341)$, тяжелый сепсис (с разной локализацией очага - брюшная полость, ЦНС, мягкие ткани и др.) (11,0 и 14,9 \%; $p=0,377)$, осложненное течение онкологического заболевания (11,6 и 16,1 \%; $p=0,328)$. Декомпенсация хронических заболеваний на фоне хронического злоупотребления алкоголем (МКБ: Ф10.Х) отмечена у 29,5 и 30,5 \% ( $p=0,942)$, ВИЧ-носительство выявлено у 2,1 и $2,3 \%$ пациентов $(p=0,815)$.

Структура инфекций нижних дыхательных путей и легкого в сравниваемых группах представлена в табл. 1.

Как видно из таблицы, клинические формы респираторных инфекций у пациентов ОАР в преинтервенционном и интервенционном периодах не претерпели существенных изменений $(p=0,244)$ и не могли оказать влияние на продолжительность АMT респираторных инфекций, ИВЛ, койко-день в ОАР и летальность.

Внедрение протокола изменило содержание АMT. В преинтервенционном периоде АМТ получали более 90 \% пациентов ОАР и практически все пациенты, которым проводилась ИВЛ через эндотрахеальную трубку или трахеостомическую канюлю, независимо от наличия или отсутствия признаков инфекции. При лечении пациентов с респираторной инфекцией основным критерием выбора антибиотика была тяжесть состояния, при этом стратификация риска антибиотикорезистентных патогенов не проводилась, а взятие материала 
Таблица 1. Структура инфекций нижних дыхательных путей и легкого в сравниваемых группах

Table 1. The structure of lower respiratory tract and lung infections in the compared groups

\begin{tabular}{|c|c|c|c|}
\hline \multirow[b]{2}{*}{ Диагноз } & \multicolumn{2}{|c|}{ Сравниваемые группы } & \multirow[b]{2}{*}{$P$} \\
\hline & $\begin{array}{c}\text { Преинтервенционный период, } \\
\qquad n=146\end{array}$ & $\begin{array}{c}\text { Интервенционный период, } \\
n=174\end{array}$ & \\
\hline Внебольничная пневмония, $n$ (\%) & $11(7,5)$ & $25(14,4)$ & 0,075 \\
\hline Нозокомиальная пневмония, не связанная с ИВЛ, $n$ (\%) & $44(30,1)$ & $54(31,0)$ & 0,903 \\
\hline Вентилятор-ассоциированная пневмония, $n$ (\%) & $85(58,2)$ & $88(50,6)$ & 0,179 \\
\hline Другие инфекции, $n$ (\%) & $6(4,1)$ & $7(4,0)$ & 0,999 \\
\hline
\end{tabular}

для бактериологического исследования выполнялось в основном если на фоне проводимой АМТ сохранялись признаки инфекционного процесса. Отмена антимикробных препаратов тоже практически не зависела от наличия признаков инфекции, пациенты в критическом состоянии продолжали ее получать даже при отсутствии инфекционного процесса, а если состояние пациента улучшалось, то терапию прекращали в момент перевода пациента в профильное отделение, при этом какого-либо обоснования отмены АМТ в медицинских картах не обнаружено.

В интервенционный период, в соответствии с принятым протоколом, АМТ респираторной инфекции начинали только в случае подтверждения инфекционного процесса в нижних дыхательных путях и легком, взятия материала для бактериологического исследования и отнесения пациента к одному из трех типов по риску наличия резистентных возбудителей. Пациентам 1-го типа, не имеющим факторов риска наличия резистентных патогенов, назначали левофлоксацин, в качестве альтернативной схемы (при известной аллергии к препарату выбора) - амоксициллин/клавуланат. В структуре возбудителей инфекции в этой группе преобладали S. pneumoniae (46 \%) и E. aerogenes $(24 \%)$, чувствительные к большинству тестируемых антимикробных препаратов. Пациентам 2-го типа с высоким риском наличия продуцентов $\beta$-лактамаз расширенного спектра (БЛРС), назначали цефоперазон/сульбактам в сочетании с амикацином, а в альтернативной схеме - меропенем (в стандартной дозировке 3 г/сутки) в сочетании с левофлоксацином. Среди патогенов у пациентов этого типа наиболее часто были верифицированы K. pneumoniae (75 \%) и E. aerogenes $(28,6 \%)$, реже $P$. aeruginosa $(14 \%)$ и $S$. aureus. Резистентность к цефалоспоринам III поколения среди патогенов, выявленных у пациентов 2-го типа, составляла 82,9 \%, к ингибитор-защищенным пенициллинам - более $47 \%$.

Пациентам 3-го типа с высоким риском полирезистентных патогенов в качестве стартовой терапии респираторных инфекций назначали меропенем в сочетании с ампициллин/сульбактамом или полимиксином, комбинируя с ванкомицином или линезолидом. По результатам бактериологических исследований в этой группе преобладали патогены из группы ESKAPE $P$. aeruginosa, Acinetobacter spp., $K$. pneumoniae, MRSA. Резистентность к карбапенемам составила 54 \%, к амикацину, цефипиму - 55 \%.

После получения результатов бактериологического исследования проводилась оптимизация АМТ: отмена антимикробных препаратов, действующих на грамположительную флору, в случае ее отсутствия - переход на антимикробные препараты узкого спектра, по воз-

Таблица 2. Влияние протокола на продолжительность курса антимикробной терапии и вероятность легочной реинфекции

Table 2. The effect of the Protocol on the duration of antimicrobial stewardship protocol and the possibility of pulmonary reinfection

\begin{tabular}{|lcc|}
\multicolumn{1}{|c|}{ Показатели } & $\begin{array}{c}\text { Преинтервенционный период, } \\
\boldsymbol{n}\end{array}$ & $\begin{array}{c}\text { Интервенционный период, } \\
\boldsymbol{n}=174\end{array}$ \\
\hline Средняя длительность курса АМТ в ОАР, дней, Ме (Q1-Q3) & $7(3-12)$ & $5(1-8)$ \\
\hline $\begin{array}{l}\text { Средняя длительность курса АМТ (ОАР + профильное } \\
\text { отделение), дней, }(\mu \pm \delta)\end{array}$ & $13,7 \pm 5,7$ & $10,4 \pm 5,8$ \\
\hline Частота легочной реинфекции, $n(\%)$ & $33(22,6)$ & $22(12,6)$ \\
\hline
\end{tabular}

АМТ - антимикробная терапия; ОАР - отделение анестезиологии и реаниматологии. 
можности - назначение монотерапии. Рекомендованная протоколом продолжительность терапии составляла 7 суток, степень выполнения этой рекомендации представлена в табл. 2.

Как видно из табл. 2, внедрение протокола эмпирической АМТ позволило значимо снизить среднюю длительность проведения АМТ в ОАР на 2 дня (с 7 до 5 дней; $p=0,034)$ и общую длительность курса (когда АМТ начинали в профильном отделении и продолжали в ОАР или назначали в ОАР и продолжали в профильном отделении) на 3 дня (с 13,7 до 10,4 дня; $p<0,01$ ). Такой результат стал возможен благодаря включению в протокол четких критериев отмены и назначения AMT, ежедневному мультидисциплинарному подходу к контролю за выполнением протокола. При этом частота рецидивов развития легочной инфекции не только не увеличилась, но и оказалась при использовании протокола существенно ниже $(22,6$ и $12,6 \%$ соответственно; $p=0,028)$, что может быть еще одним признаком эффективности применения протокола. Согласно данным современных исследований для уменьшения микробной массы ниже критического уровня достаточно 7 суток эффективной АМТ [16]. Реализация алгоритма диагностики и адресной АМТ, проводимой с учетом локальных данных о наиболее вероятных возбудителях инфекции и их свойствах, повышает эффективность лечения и тем самым позволяет обоснованно, без опасности для пациента раньше прекратить введение антимикробных препаратов. Необоснованно длительное применение антибиотиков приводит к появлению и распространению резистентных микроорганизмов, развитию у больных новых нозокомиальных суперинфекций, аллергических и/или токсических реакций. В конечном итоге это ухудшает состояние пациента и снижает эффективность лечения.

Результаты лечения пациентов с респираторными инфекциями в ОАР представлены в табл. 3.

Таблица 3. Влияние протокола антимикробной терапии на результаты лечения респираторных инфекций в ОАР

Table 3. The Impact of antimicrobial stewardship protocol on Respiratory Infection Treatment Results in ICU

\begin{tabular}{|c|c|c|c|}
\hline Показатели & $\begin{array}{c}\text { Преинтервенционный период, } \\
n=146\end{array}$ & $\begin{array}{c}\text { Интервенционный период, } \\
n=174\end{array}$ & $P$ \\
\hline Продолжительность лечения в ОАР, сутки, Ме (Q1-Q3) & $10(3-20,5)$ & $7(1,25-14)$ & 0,025 \\
\hline Пациенты с инфекцией, переведенные на ИВЛ* , $n$ (\%) & $32(52,5 \%)$ & $40(46,5 \%)$ & 0,587 \\
\hline Средняя продолжительность ИВЛ, сутки, Ме (Q1-Q3) & $6(1,0-14,75)$ & $3(0,5-11,75)$ & $<0,01$ \\
\hline Пациенты, нуждавшиеся в ИВЛ > 48 ч** , $n$ (\%) & $30(93,75)$ & $29(72,5)$ & 0,044 \\
\hline $\begin{array}{l}\text { Пациенты, нуждавшиеся в проведении ИВЛ > } 21 \text { суток, } \\
n(\%)\end{array}$ & $19(16,2)$ & $17(13,3)$ & 0,637 \\
\hline Число летальных исходов в сравниваемых группах, $n$ (\%) & $69(47,2)$ & $63(36,2)$ & 0,06 \\
\hline
\end{tabular}

* Без учета пациентов с вентилятор-ассоциированной пневмонией (см. табл. 1).

** Доля пациентов от переведенных на искусственную вентиляцию легких на фоне инфекции нижних дыхательных путей и легких (см. вторую строку табл.).

Как видно из табл. 3, внедрение протокола не привело к статистически значимому снижению летальности в сравниваемых группах, частоте переводов пациентов на ИВЛ на фоне уже развившегося инфекционного процесса, а также к снижению доли пациентов, которым респираторная поддержка была необходима более 21 суток. Это объяснимо, поскольку эти показатели зависят от многих факторов. У пациентов в критическом состоянии даже незначительные воспалительные изменения, вызванные инфекционным процессом в легком, могут привести к утрате последних резервов и декомпенсации функции внешнего дыхания, способствуя в ряде случаев развитию необратимых нарушений или фатальных осложнений. Конечно, вероятность такого неблагоприятного развития событий в определенной степени зависит от того, как быстро была диагностирована инфекция и начата эффективная стартовая терапия, но важное значение имеют и многие другие обстоятельства, и в первую очередь - причина поступления в ОАР и тяжесть коморбидных нарушений. В нашем исследовании респираторные инфекции развились у пациентов, госпитализированных более чем по 15 профилям оказания медицинской помощи с самыми разнообразными нозологическими формами заболеваний. В условиях ОАР многопрофильного стационара выполнить атрибутивный анализ и выделить влияние протокола на результаты лечения таких пациентов сложно, и на данном этапе исследования такие попытки оказались малоинформативными. Поэтому представленные в табл. 3 данные о летальности имеют обобщенный характер и не отражают летальность от респираторных инфекций в ОАР, они в первую очередь зависят от специфики основного заболевания или травмы. На основании полученных результатов можно лишь отметить незначительную положительную тенденцию в снижении потребности в респираторной поддерж- 
ке и снижении летальности и подтвердить, что внедрение протокола с определенными ограничениями по продолжительности АМТ не привело к ухудшению результатов лечения пациентов в критическом состоянии.

Однако следует отметить, что в интервенционном периоде отмечены и существенные положительные изменения значений исследуемых показателей: в среднем на трое суток сократилась продолжительность пребывания пациентов с респираторной инфекцией в ОАР $(p=0,025)$ и продолжительность ИВЛ $(<0,01)$; а среди пациентов, переведенных на ИВЛ, существенно с 6,25 до 27,5 \% ( $p=0,044)$ - увеличилась доля пациентов, которым респираторная поддержка требовалась менее 48 ч. На наш взгляд, эти изменения как раз и отражают эффективность терапии, проводимой в первые дни развития инфекции, которая обеспечила более быстрое улучшение состояния пациента, восстановление самостоятельного дыхания с сокращением продолжительности и потребности в ИВЛ, позволила раньше начать активизацию пациента и продолжить его лечение в условиях профильного отделения. Эти результаты мы считаем следствием внедрения протокола в повседневную практику и контроля за выполнением его положений.

\section{Заключение}

Создание и внедрение в повседневную практику локального протокола лечения респираторных инфек- ций в ОАР многопрофильного стационара являются действенным инструментом контроля АМТ. Выполнение положений протокола позволяет врачу своевременно и обоснованно выбрать и назначить каждому пациенту те антимикробные препараты, которые с максимальной вероятностью обеспечат раннее начало эффективной терапии, а затем также обоснованно и своевременно изменить схему или прекратить терапию. В конечном итоге улучшаются результаты лечения, снижается вероятность прогрессирования или рецидива инфекции, сокращаются длительность респираторной поддержки и общая продолжительность пребывания пациентов в ОРИТ.

Конфликт интересов. Авторы заявляют об отсутствии конфликта интересов.

Вклад авторов. Замятин М.Н., Векшина О.А., Гусаров В.Г., Кузьминов С.У., Бардин И.В., Князева П.А. - разработка концепции статьи, получение и анализ фактических данных, написание и редактирование текста статьи, проверка и утверждение текста статьи.

\section{ORCID авторов}

Замятин М.Н. - 0000-0002-2072-7798

Векшина О.А. - 0000-0002-4988-3698

Гусаров В.Г. - 0000-0002-2900-1459

Кузьминов С.У. - 0000-0001-5456-2162

Бардин И.В. - 0000-0001-6870-8791

Князева П.А. - 0000-0002-2317-966Х

\section{Литература/References}

[1] Нозокомиальная пневмония у взрослых: Российские национальные рекомендации. Под ред. акад. РАН Б.Р. Гельфанда; отв. ред. к. м. Н., доцент Д.Н. Проценко, к. м. н., доцент Б.3. Белоцерковский. 2-е изд., перераб. и доп. М.: Медицинское информационное агентство, 2016. [Nosocomial pneumonia in adults: Russian National guidelines. Gelfand B.R. Moscow: MIA, 2016. (In Russ)]

[2] Яковлев С.В., Суворова М.П., Белобородов В.Б. и др. Распространенность и клиническое значение нозокомиальных инфекций в лечебных учреждениях России: исследование ЭРГИНИ. Антибиотики и химиотерапия. 2016; 61(5-6): 32-42. [Yakovlev S.V., Suvorova M.P., Beloborodov V.B., etal. Multicenter Study of the Prevalence and Clinical value of Hospital-Acquired Infections of Emergency Hospitals in Russia: ERGINI Study Team. Antibiot Khimioter. 2016; 61(5-6): 32-42. (In Russ)]

[3] Chastre J., Fagon J.Y. Ventilator-associated pneumonia. Am J Respir Crit Care Med. 2002; 165(7): 867-903. Review.

[4] Welte T., Torres A., Nathwani D. Clinical and economic burden of community-acquired pneumonia among adults in Europe. Thorax. 2012; 67(1): 71-79.
[5] Torres A., Zhong N., Pachl J., et al. Ceftazidime-avibactam versus meropenem in nosocomial pneumonia, including ventilatorassociated pneumonia (REPROVE): a randomised, double-blind, phase 3 non-inferiority trial. Lancet Infect Dis. 2017; pii: S14733099(17)30747-8

[6] KollefM.H., Nováček M., Kivistik Ü., et al. Ceftolozane-tazobactam versus meropenem for treatment of nosocomial pneumonia (ASPECT-NP): a randomised, controlled, double-blind, phase 3, non-inferiority trial. Lancet Infect Dis. 2019; 19(12): 1299-1311. DOI: 10.1016/S1473-3099(19)30403-7. Epub 2019 Sep 25.

[7] Ferrer R., Martin-Loeches I., Phillips G., et al. Empiric antibiotic treatment reduces mortality in severe sepsis and septic shock from the first hour: results from a guideline-based performance improvement program. Crit Care Med. 2014; 42(8): 1749-1755.

[8] Стратегия и тактика применения антимикробных средств в лечебных учреждениях России: Российские национальные рекомендации. Под ред. Савельева В.С., Гельфанда Б.В., Яковлева С.В. М.: Компания Боргес, 2012. [Antimicrobial Stewardship Strategy in Russian Hospital: Russian National Guidelines. Savelev V.S., Gelfand B.V., Yakovlev S.V. - Moscow: Company Borges, 2012. (In Russ)] 
[9] Программа СКАТ (Стратегия контроля антимикробной терапии) при оказании стационарной медицинской помощи: Российские клинические рекомендации. Под ред. Яковлева С.В., Брико Н.И., Сидоренко С.В., Проценко Д.Н. М. Перо, 2018. [Antimicrobial Stewardship Strategy (AMS strategy): The Clinical Guidelines. Yakovlev S.V., Briko N.I., Sidorenko S.V., Protsenko D.N. Moscow, 2018. (In Russ)]

[10] Левит А.Л., Сидоренко С.В., Яковлев В.П. и др. Анализ адекватности стартовых эмпирических режимов антибактериальной терапии при тяжелых нозокомиальных инфекциях (исследование АСЭТ). Клиническая фармакология и терапия. 2006: 2; 21-14. [Levit A.L., Sidorenko S.V., Yakovlev V.P., et al. Adequacy and efficacy of initial empiric antibiotic treatments in severe nosocomial infections in ICU departments: results of multicentre randomised study. Clinical pharmacology and therapy. 2006: 2; 21-14. (In Russ)]

[11] Arnold H.M., Micek S.T., Skrupky L.P., Kollef M.H. Antibiotic stewardship in the intensive care unit. Semin Respir Crit Care Med. 2011; 32: 215-227.

[12] Leuthner K.D., Doern G.V. Antimicrobial stewardship programs. J Clin Microbiol. 2013; 51: 3916-3920.
[13] Гусаров В.Г. Замятин М.Н., Теплых Б.А Антибиотикорезистентность: пути решения проблемь в многопрофильном стационаре. Вестник Национального медико-хирургического центра им. Н.И. Пирогова. 2014; 9(3): 108-113. [Gusarov V.G., Zamjatin M.N., Teplyh B.A. Antibiotic Resistance: Possible Solutions In A Multidisciplinary Hospital. Bulletin of Pirogov National medical and surgical center. 2014 9(3): 108-113. (In Russ)]

[14] CDC. Core Elements of Hospital Antibiotic Stewardship Programs. Atlanta, GA: US Department of Health and Human Services, CDC; 2019. https://www.cdc.gov/antibiotic-use/coreelements/hospital.html

[15] Руднов В.А., Колотова Г.Б., Багин В.А. и др. Управление антимикробной терапией в службе реанимации и интенсивной терапии КМАХ. 2018; 20(2): 132-140. DOI: 10.36488/cmac.2018.2.132-140. [Rudnov V.A., Kolotova G.A., Bagin V.A., et al. The role of antimicrobial therapy stewardship in intensive care service. J Clinical microbiology and antimicrobial chemotherapy. 2018; 2: 132-140. (In Russ)]

[16] Pugh R., Grant C., Cooke R.P., et al. Short-course versus prolongedcourse antibiotic therapy for hospital-acquired pneumonia in critically ill adults. Cochrane Database Syst Rev. 2015; 24(8): CD007577. 